\title{
Trans-inhibition of HIV-I by a long hairpin RNA expressed within the viral genome Pavlina Konstantinova ${ }^{1}$, Olivier ter Brake ${ }^{1}$, Joost Haasnoot ${ }^{1}$, Peter de Haan ${ }^{2}$ and Ben Berkhout*1
}

Address: ${ }^{1}$ Laboratory of Experimental Virology, Department of Medical Microbiology, Center for Infection and Immunity Amsterdam (CINIMA), Academic Medical Center, University of Amsterdam, Meibergdreef 15, 1105 AZ Amsterdam, The Netherlands and ${ }^{2}$ Viruvation B. V. Wassenaarseweg 72, 2333 AL Leiden, The Netherlands

Email: Pavlina Konstantinova - p.s.konstantinova@amc.uva.nl; Olivier ter Brake - o.terbrake@amc.uva.nl; Joost Haasnoot - p.c.haasnoot@amc.uva.nl; Peter de Haan - peter.dehaan@phytovation.nl; Ben Berkhout* - b.berkhout@amc.uva.nl

* Corresponding author

Published: I March 2007

Retrovirology 2007, 4:15 doi:10.1186/1742-4690-4-15

This article is available from: http://www.retrovirology.com/content/4/I/15

(c) 2007 Konstantinova et al; licensee BioMed Central Ltd.

This is an Open Access article distributed under the terms of the Creative Commons Attribution License (http://creativecommons.org/licenses/by/2.0), which permits unrestricted use, distribution, and reproduction in any medium, provided the original work is properly cited.

\begin{abstract}
Background: Human immunodeficiency virus type I (HIV-I) can be inhibited by means of RNA silencing or interference (RNAi) using synthetic short interfering RNAs (siRNAs) or gene constructs encoding short hairpin RNAs (shRNAs) or long hairpin RNAs (IhRNAs). The use of siRNA and shRNA as antiviral therapeutic is limited because of the emergence of viral escape mutants. This problem is theoretically prevented by intracellular expression of IhRNAs generating multiple siRNAs that target the virus simultaneously, thus reducing the chance of viral escape. However, gene constructs encoding IhRNA molecules face problems with delivery to the right cells in an infected individual. In order to solve this problem, we constructed an HIV-I variant with a 300 bp long hairpin structure in the 3' part of the genome corresponding to the Nef gene (HIVIhNef).
\end{abstract}

Results: Intriguingly, HIV-IhNef potently inhibited wild-type HIV-I production in trans. However, HIV-IhNef demonstrated a severe production and replication defect, which we were able to solve by selecting spontaneous virus variants with truncated hairpin structures. Although these escape variants lost the ability to trans-inhibit HIV-I, they effectively outgrew the wild-type virus in competition experiments in SupTI cells.

Conclusion: Expression of the IhNef hairpin within the HIV-I genome results in potent transinhibition of wild-type HIV-I. Although the mechanism of trans-inhibition is currently unknown, it remains of interest to study the molecular details because the observed effect is extremely potent. This may have implications for the development of virus strains to be used as live-attenuated virus vaccines.

\section{Background}

RNA interference (RNAi) has been used to inhibit the replication of a wide range of viruses including the human immunodeficiency virus type 1 (HIV-1), hepatitis $\mathrm{C}$ virus
(HCV), hepatitis B virus (HBV), dengue virus, poliovirus, influenza virus A, coronaviruses, herpesviruses, and picornaviruses $[1,2]$. Due to its sequence specificity, RNAi is a potentially selective method for intracellular immuniza- 
tion against HIV-1 infection. RNAi-mediated suppression of HIV-1 replication has been accomplished by synthetic small interfering RNAs (siRNAs) in a transient manner [36] and by shRNA expression vectors in stably transfected cells [7-9]. Despite potent inhibition, the use of both approaches as therapeutic antiviral is limited because of the rapid emergence of HIV-1 escape mutants [9-11]. Strategies to reduce the chance of viral escape include the simultaneous use of multiple siRNAs $[12,13]$, the intracellular expression of a second generation of escape-anticipating shRNAs [14], or microRNA-based double-stranded RNAs (miRNAs), which do not require perfect sequence complementarity for inhibition $[15,16]$.

An alternative method to inhibit HIV-1 is the use of gene constructs encoding HIV-1-specific long hairpin RNAs (lhRNAs, transcripts folding an extended hairpin structure) or long double-stranded RNAs (dsRNAs, two complementary transcripts that form an extended duplex). These molecules should yield multiple effective siRNAs upon intracellular processing $[6,17,18]$. However, lhRNA approaches raise concerns about induction of the dsRNAtriggered interferon (IFN) response. Others and we have shown that endogenously expressed lhRNA and dsRNA can inhibit HIV-1 production without induction of the innate antiviral response [18-21]. In fact, most reports on IFN induction by long dsRNAs in mammalian cells are based on transfection of cells with in vitro synthesized dsRNAs [22,23]. Apparently, endogenously produced dsRNA is less active than exogenous dsRNA in inducing the IFN response.

Several antiviral approaches using extended lhRNA and long dsRNA molecules have been reported in plant and insect cells that lack the innate antiviral IFN response. Transient expression of DNA constructs encoding virusspecific dsRNA in plant protoplasts or insect cells partially protects the cells from infection by the homologous virus $[23,24]$. Stable expression of such constructs renders the cells resistant to infection $[25,26]$. lhRNA can inhibit HIV1 production under certain conditions, without induction of the IFN response $[6,17,18]$. Ideally, a single lhRNA should generate multiple effective siRNAs upon intracellular processing, providing more durable inhibition of HIV-1 than a single shRNA. An additional advantage of lhRNA inhibitors is that it does not require pre-determination of the optimal shRNAs and corresponding HIV-1 target sequences because multiple effective siRNAs will be produced. A potential disadvantage of the use of lhRNA as therapeutic is that the generation of multiple siRNAs will be more likely to cause off-target effects.

We have previously reported strong inhibition of HIV-1 production using gene constructs encoding HIV-specific lhRNAs and dsRNA in transient transfection assays [18].
An alternative for stable expression of shRNAs is a conditionally replicating HIV-1-based virus, which was previously used by us to deliver an antiviral shRNA cassette into HIV-1 susceptible target cells [27]. In the current study, we constructed the HIV-lhNef variant, which contains a 300 bp extended hairpin structure at the 3 ' genome position of the Nef gene of the otherwise wild-type HIV-1. We tested this HIV-lhNef for its capacity to inhibit the production of wild-type virus. Intriguingly, HIV-lhNef potently inhibited wild-type HIV-1 production in trans. However, HIV-lhNef demonstrated a severe production and replication defect, which we were able to solve by selecting spontaneous, escape viruses.

\section{Results}

\section{The HIV-lhNef variants}

We previously described the construction of the HIVlhNef virus variant [28], which contains a 300 bp extended hairpin structure (Fig. 1A). The hairpin structure is present in the full-length genomic RNA and all spliced mRNAs. This structure induced a severe virus production and replication defect. Similarly, we were not able to obtain stable expression of the lhRNA inhibitor from a lentiviral vector (results not shown), probably due to problems in reverse transcription of the excessively stable hairpin structure [29]. The presence of the lhNef insert resulted in a dramatic drop of the viral transduction titer, possibly also due to self-targeting of Nef/LTR sequences in the vector genome. Unlike the non-replicating lentiviral vector, the HIV-lhNef virus could generate spontaneous variants by evolution. Indeed, replicating virus variants could be selected with a severely truncated lhNef hairpin structure [28]. These escape variants are listed in Figure $1 \mathrm{~B}$, with the number of basepairs in the remaining hairpin structure in their name. For instance, AS44 has 44 remaining basepairs, producing a hairpin of intermediate stability $(\Delta \mathrm{G}=-84.7 \mathrm{kcal} / \mathrm{mol})$. We now set out to test these variants in further detail, e.g. for their ability to inhibit wild-type HIV-1 in trans.

\section{Trans-inhibition of wild-type HIV-I}

HEK293T cells were transfected with the wild type HIV-1 construct or HIV-lhNef. We measured CA-p24 in the culture supernatant as a measure of virus production 2 days post-transfection. Whereas wild-type HIV-1 produced high CA-p24 levels, no virus production was detected for HIV-lhNef (Fig. 2A, upper panel). This loss of virus production is due to the presence of the lhNef hairpin because no such effect was scored for several control constructs: HIV-1 with a CMV insertion in sense and antisense orientation (HIV-CMV and HIV-asCMV) and the Nefdeleted HIV-1 construct R1 (Fig. 2A). Notably, HIVasCMV did not produce high CA-p24 values probably due to promoter interference from the strong CMV promoter. We next co-transfected equal amounts of wild-type HIV-1 


\section{A}

\section{HIV-IhNef mutant}

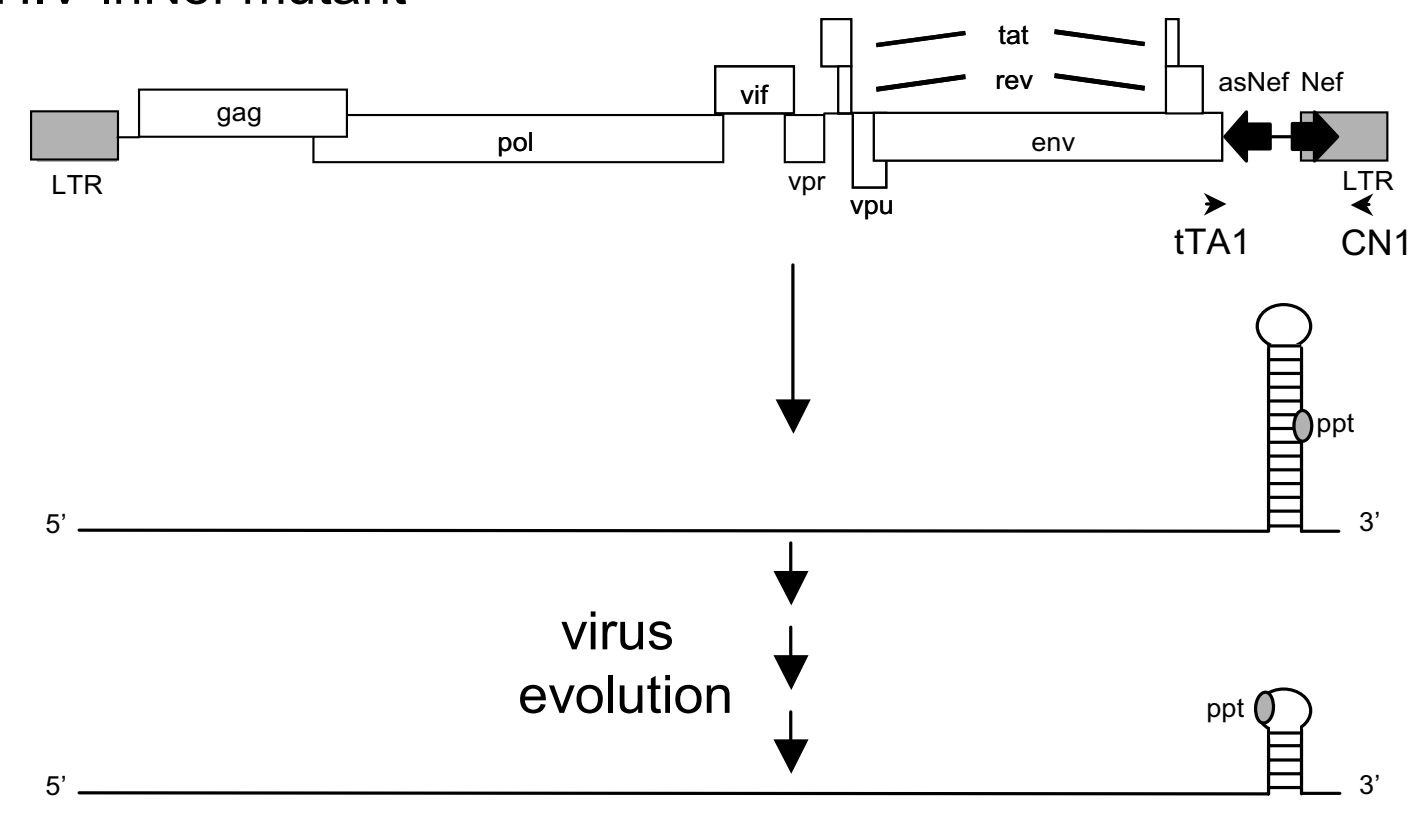

B

\section{$\Delta \mathrm{G}(\mathrm{kcal} / \mathrm{mol}) \quad$ replication}

$\begin{array}{rcc}\text { HIV-IhNef mutant } & -648.2 & - \\ \text { escape variants: AS44 } & -84.7 & + \text { - } \\ \text { AS15 } & -23.1 & + \\ \text { AS19 } & -31.8 & + \\ \text { AS11 } & -16.7 & + \\ \text { AS8 } & -7.5 & +\end{array}$

\section{Figure I}

The HIV-IhNef RNA genome encodes an extended hairpin that is truncated by virus evolution. (A) The proviral DNA genome, highlighting the insertion of the antisense asNef fragment (original Nef coordinates +8544 to +8844 ). The primers tTAI and $\mathrm{CNI}$ used to amplify the inverted repeat region are indicated. The inverted repeat (thick arrows) results in the formation of a 300 bp hairpin structure in the RNA genome of HIV-lhNef (middle panel). This hairpin encompasses the polypurine tract (ppt). The truncated hairpin structure that emerged by virus evolution is shown in the lower panel. (B) HIV-lhNef escape variants were previously described in detail [28]. The name AS (antisense) reflects the number of basepairs in the remaining RNA hairpin. $\Delta \mathrm{G}$ is the thermodynamic stability of the remaining perfectly basepaired stem segment. The replication column shows the replication capacity of the viruses. 
with HIV-lhNef, HIV-CMV, HIV-asCMV or R1 (Fig. 2A, lower panel). Interestingly, only HIV-lhNef was able to potently inhibit wild-type HIV-1 production in trans. The level of inhibition is comparable to that obtained in a cotransfection with the highly effective shNef inhibitor [9], which we used as a positive control. We next titrated the HIV-lhNef construct to test if the inhibitory effect is concentration-dependent (Fig. 2B). We measured 92\% inhibition of wild-type virus production when mixed 2:1 with the HIV-lhNef inhibitory construct. Even at a 7:1 ratio, HIV-1 production was reduced by $61 \%$. The mechanism of this potent inhibition is currently unknown. Although HIV-lhNef may be a potently interfering construct, a major problem is that it does not replicate.

\section{Replication properties of the escape viruses}

We studied the replication potential of HIV-lhNef and the AS escape variants in PBMC (Fig. 3). As a positive control, the Nef-positive wild-type HIV-1 construct was used. As shown previously, HIV-lhNef did not replicate to detectable levels. The escape variant AS44, with the intermediate length hairpin, replicated only marginally. All other AS variants replicated efficiently (results are summarized in Figure 1B), although these variants reached maximal CAp24 values at least 1 log lower than that of wild-type HIV1. This phenotypic difference can be explained by the Nefminus genotype of these viral strains as the accessory Nef protein contributes to efficient virus replication in primary cells $[30,31]$.

We next transfected the plasmid constructs encoding wildtype HIV-1, HIV-lhNef and the AS escape variants in HEK293T cells. These cells do not support HIV-1 replication, but produce virus particles upon DNA transfection. Virus production was measured by CA-p24 ELISA in the culture supernatant at three days post-transfection. Unlike the original HIV-lhNef mutant, which demonstrated a severe CA-p24 production defect, all AS variants efficiently produced virus (Fig. 4A). This result demonstrates that truncation of the lhNef hairpin overcomes the production and replication defect. We next tested if the AS variants are able to inhibit wild-type HIV-1 in trans in the co-transfection assay. Compared to the effective HIVlhNef inhibitor, all AS escape variants had lost the ability to actively inhibit wild-type virus production (Fig. 4B). Even the poorly replicating AS44 variant lost the capacity to inhibit HIV-1 in trans. Thus, the capacity of HIV-lhNef to inhibit HIV-1 correlates with its replication defect.

\section{Virus competition between HIV-I and the HIV-IhNef escape variants}

As a more sensitive assay for possible trans-inhibition, we tested the individual AS variants in a direct competition with wild-type HIV-1 in PBMC. Cells were infected with an equimolar mixture of the two viruses (based on CA- p24) that were produced in HEK293T cells, and virus was passaged to fresh cells at the peak of infection. Virus replication was monitored by CA-p24 ELISA and visual inspection for syncytia. Cellular DNA was extracted at several times post-infection and the proviral Nef region was PCR amplified with primers tTA1 and CN1 (see Fig. 1). Since the PCR products will differ in size for wild-type HIV-1 and each AS variant, both competitors can be detected in the same sample by subsequent agarose gel electrophoresis of the DNA fragments. The outgrowth of a particular virus was verified by cloning and sequencing of the PCR products from the last time-point sample (results not shown).

Wild-type HIV-1 effectively outcompeted all AS mutants in PBMC. An example of the competition between HIV-1 and the AS19 variant is provided in Figure 5A. Both viruses are detected in the culture at day 5, but we observed a gradual increase in the intensity of the larger PCR product. A single PCR product was observed at day 58 , indicating that HIV-1 predominated the culture and thus effectively outcompeted AS19. The same result was obtained when HIV-1 and AS19 were mixed in a 1:10 ratio (Fig. 5B). The competition results for all AS variants are summarized in Table 1 and shown in Additional file 1. The AS variants are likely to be less replication competent than wild-type HIV-1 due to the Nef-minus genotype, but the remnant hairpin structure may also impose a negative effect on the replication capacity. We therefore included the unrelated Nef-minus mutant R1 with a 106 nt deletion in Nef, but without a hairpin structure [9]. R1 produces CA-p24 levels comparable to wild-type HIV-1 and cannot inhibit viral production in trans (Fig. 2 and 4 ). We performed competition experiments between HIV-1 and R1, but also with AS19 and R1. HIV-1 also outcompetes the alternative Nef-minus R1 mutant (Fig. 5C). AS19 and $\mathrm{R} 1$ co-existed in the culture up to 62 days post-infection (Fig. 5C), indicating that both Nef-minus viruses have very similar replication fitness.

We repeated the competition experiments in the SupT1 T cell line. Because the Nef protein has no impact on viral replication fitness in $\mathrm{T}$ cell lines [30], this system may allow a more sensitive screen for trans-inhibition of wildtype HIV-1 by the hairpin-containing AS variants. In fact, all AS mutants outcompeted wild-type HIV-1, as illustrated for the AS19 variant (Fig. 6A). The results for all AS variants are summarized in Table 1 and shown in Additional files 1, 2 and 3. Intriguingly, variant R1 co-existed with the wild-type virus for 62 days (Fig. 6B). This result confirms that the Nef function is not important in $\mathrm{T}$ cell lines. However, because the AS variants were able to outcompete HIV-1, it raises the interesting possibility of hairpin-mediated (trans) inhibition by these AS variants. Perhaps even more surprisingly, AS19 and R1 co-existed 
A

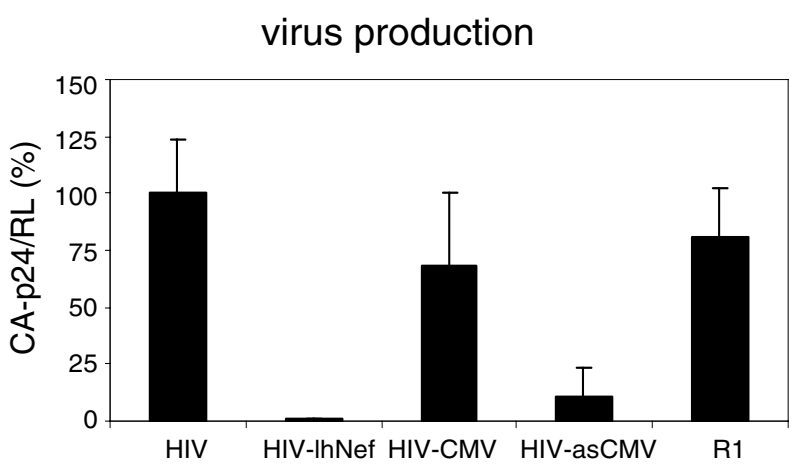

trans-inhibition of HIV-1

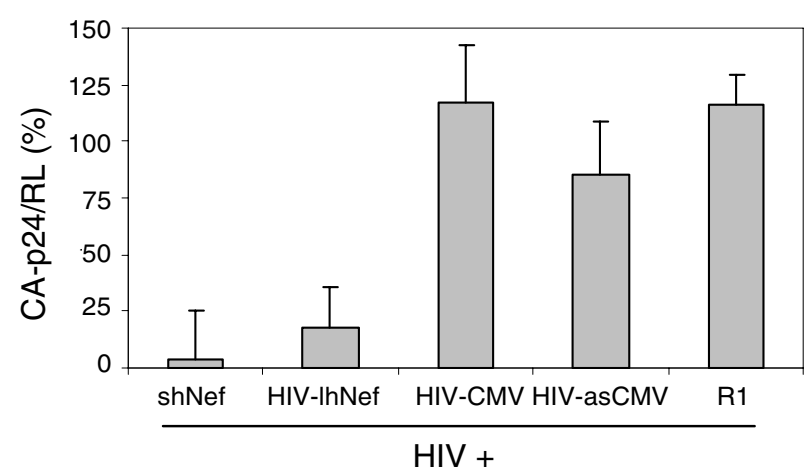

B

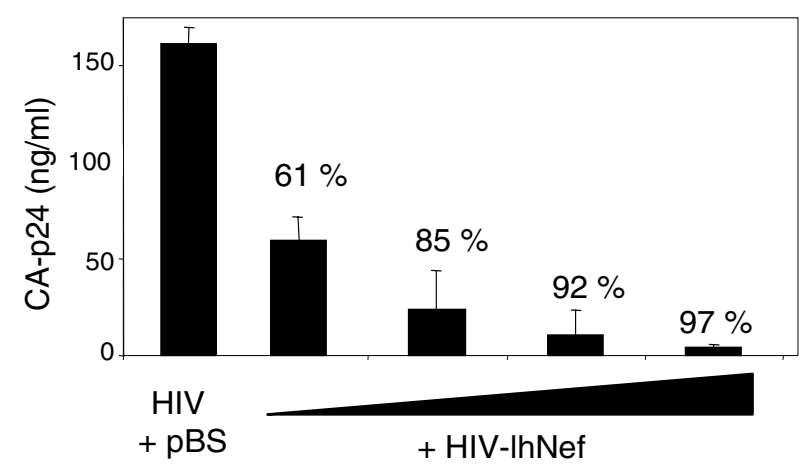

Figure 2

Trans-inhibition of HIV-I by HIV-IhNef. (A) HEK239T cells were co-transfected with I50 ng of the HIV-I molecular clone LAI, HIV-IhNef, HIV-CMV, HIV-asCMV or RI and I50 ng pBluescript (Promega) (upper panel). In the lower panel I50 ng HIV was co-transfected with I50 ng pSuper-shNef, HIV-IhNef, HIV-CMV, HIV-asCMV or RI. I ng pRL plasmid, expressing Renilla luciferase from the CMV promoter was added as an internal control for cell viability and transfection efficiency. Transfections were performed with lipofectamine- 2000 and $1.5 \times 10^{5}$ cells. Virus production was measured in the culture supernatant 2 days after transfection by CA-p24 ELISA and Renilla expression was measured with the Renilla luciferase assay system (Promega). We plotted the relative percentage of CA-p24/RL, with the HIV + pBluescript transfection set at 100\%. Error bars represent the standard deviation from quadruple transfections in three independent experiments. (B) Titration of the HIV-lhNef inhibitor. $500 \mathrm{ng}$ HIV-I was co-transfected with increasing amounts (0-75-I25-250-500 ng) of HIV-IhNef. The total DNA concentration was kept constant by adding pBluescript. Virus production was measured in the culture supernatant 2 days after transfection. Error bars represent the standard deviation in three replicates. This is a representative figure from three transfection experiments with similar results. 


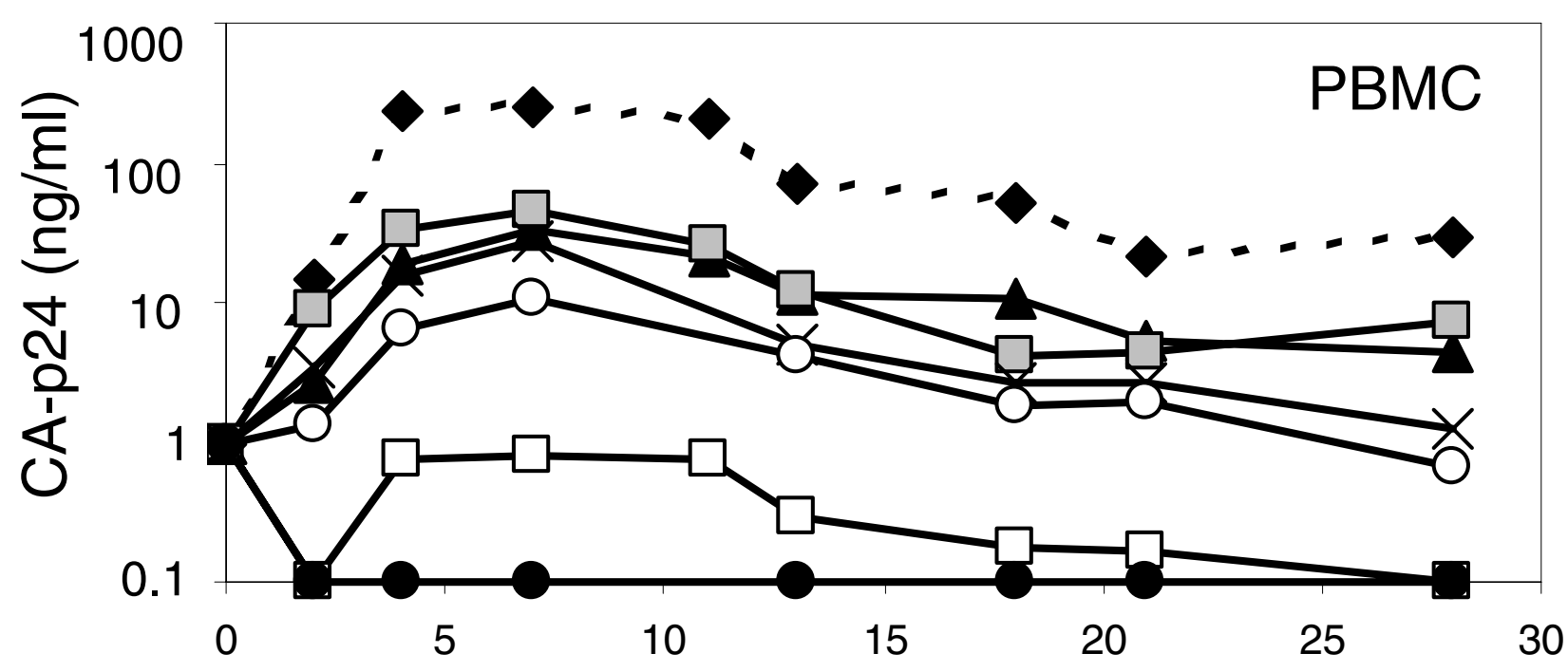

days after transfection

\section{- hIV $\square$ AS44 $\triangle A S 19 \square$ AS15 XAS11 O AS6 OHIV-lhNef}

Figure 3

Virus replication of HIV-IhNef and the escape variants in PBMC. Cells $\left(5 \times 10^{6}\right)$ were transfected with $10 \mu \mathrm{g}$ of the proviral DNA constructs and CA-p24 production was measured in the culture supernatant at several days post-transfection for up to 4 weeks. Half of the culture medium was replaced with fresh complete RPMI medium with IL-2 every 4 days and freshly activated PBMC $\left(2 \times 10^{6}\right)$ were added at every $2^{\text {nd }}$ addition. The experiment has been repeated five times for AS 19 and two times for the other variants.

for the length of the competition experiment. We speculate that the deletion mutant R1 may lack target sequences for trans-inhibition by AS19.

\section{Mechanism of trans-inhibition by HIV-IhNef and the AS variants}

We have previously described strong inhibition of HIV-1 by RNAi-inducing expression vectors encoding shRNA or lhRNA molecules directed against viral genes $[9,13,14,18]$. One possibility is that the mechanism of HIV-1 inhibition by HIV-lhNef or the AS mutants could be RNAi-related. One of the hallmarks of RNAi is its sequence-specificity. We therefore tested if HIV-lhNef could inhibit the Luc-Nef reporter, in which a 250 nt Nef target sequence was placed downstream of the Photinus luciferase gene [11]. As a positive control, we showed that HIV-lhNef induced a dramatic decrease of wild-type HIV1 production in trans, comparable in efficiency with the highly effective RNAi-inducers shGag and shNef [13] (Fig. 7A). However, no sequence-specific inhibition of the LucNef reporter was obtained with HIV-lhNef (Fig. 7B). The shGag molecule serves as a negative control in this experiment, and shNef as a potent positive control. In these transient transfection experiments a Renilla luciferase expression plasmid was included, which provides a control for transfection efficiency and possible aspecific effects. Renilla luciferase expression was similar in all transfections (results not shown), indicating that HIV- 


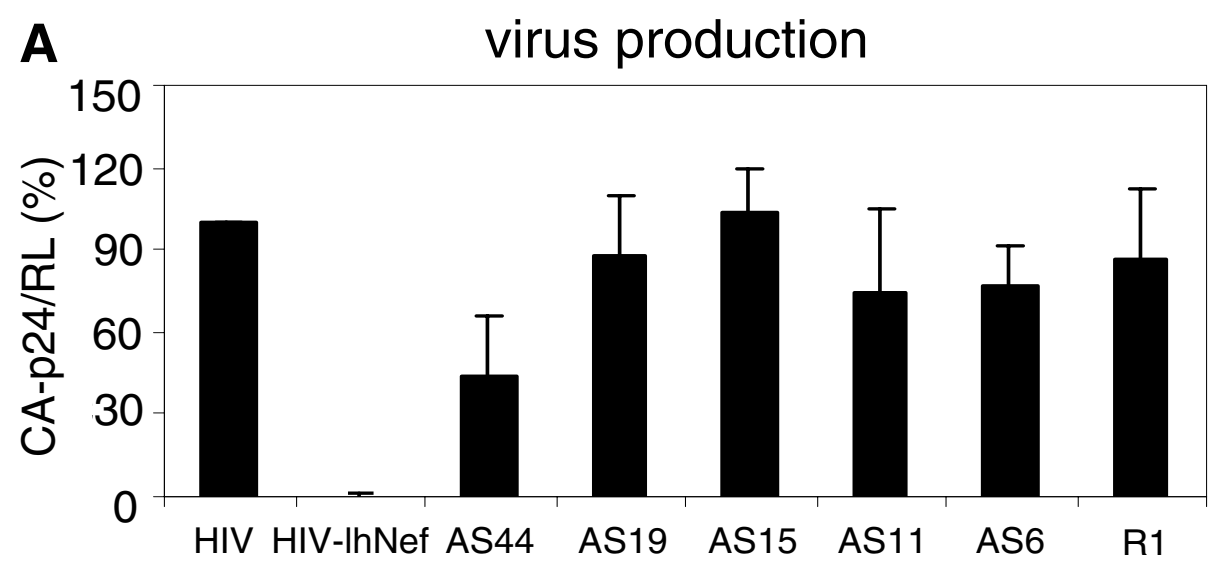

B

trans-inhibition of HIV-1

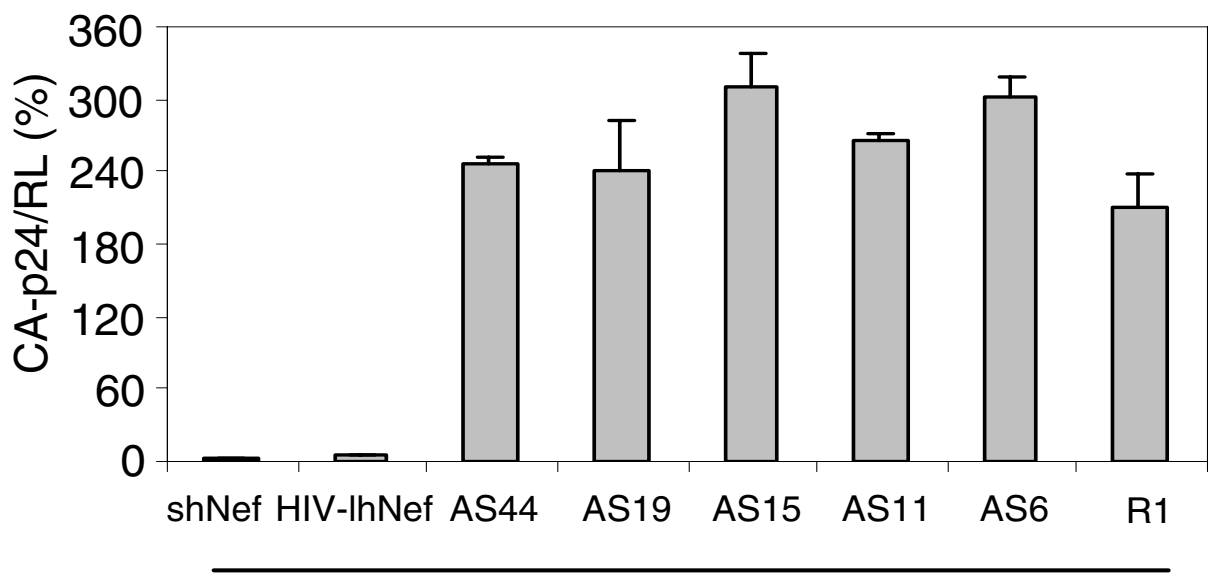

HIV +

Figure 4

Virus production of the HIV-lhNef escape variants and trans-inhibition of HIV-I. (A) Virus production in HEK293T cells. Cells were transfected with $150 \mathrm{ng}$ pBluescript and $150 \mathrm{ng}$ of the indicated constructs. I ng pRL plasmid was added as an internal control. Transfections were performed with lipofectamine- 2000 and $1.5 \times 10^{5}$ cells. Virus production was measured in the culture supernatant 2 days after transfection by CA-p24 ELISA and RL expression was measured with the Renilla luciferase assay system (Promega). We plotted the relative percentage of CA-p24/RL, with the transfection HIV + pBluescript set at I00\%. Error bars represent the standard deviation from quadruple transfections in two independent experiments. (B) Trans-inhibition of HIV-I production by the AS escape mutants. HEK293T cells were co-transfected with I50 ng HIV-I and I50 ng pSupershNef, HIV-lhNef, the AS escape mutants or RI. CA-p24 production was measured in the culture supernatant at two days post-transfection. Error bars represent the standard deviation from quadruple transfections in two independent experiments. 

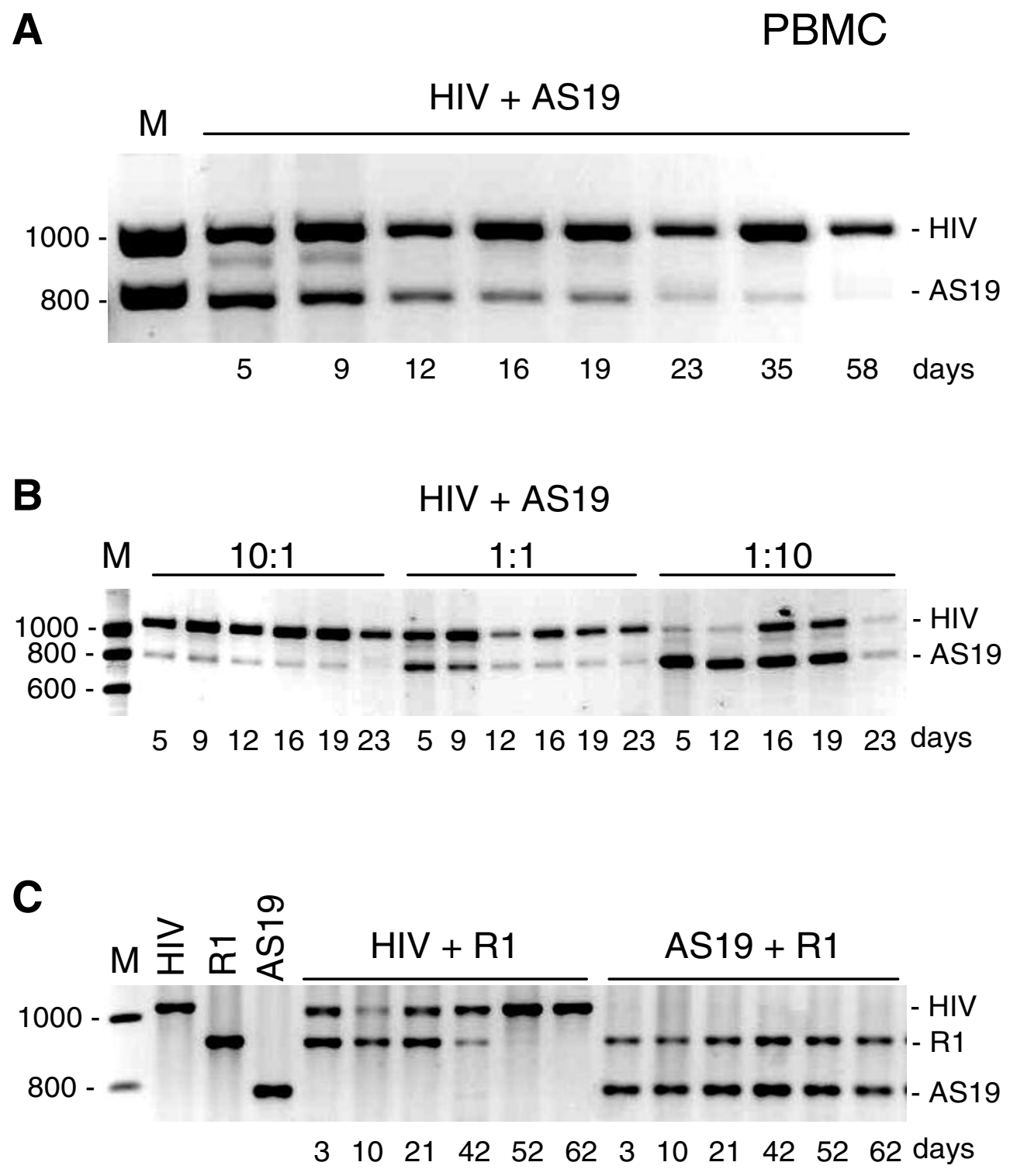

Figure 5

Virus competition experiments in PBMC. (A) Two viruses were mixed as indicated on top of the panels, usually in a I:I ratio (unless indicated otherwise, see panel B). The composition of the virus mixture was followed by PCR across the Nef region with primers tTAI and CNI (see Fig. IA). The experiment has been repeated 5 times for ASI9. Appropriate markers and molecular weight standard $(M)$ are indicated. The identity of the respective PCR fragments is indicated on the right. (B) Competition of HIV-I and ASI 9 mixed in three ratios: I0:I, I:I, I:I0. (C) Competition between the RI variant and either HIV-I or ASI9. RI is a $106 \mathrm{nt}$ Nef deletion mutant. The experiment has been repeated twice, with similar results. 
Table I: Pairwise virus competition experiments

\begin{tabular}{lll}
\hline Virus mix ${ }^{a}$ & PBMC winner & SupTI winner \\
\hline HIVIAS44 & HIV $(4)^{\mathrm{b}}$ & HIV $(4)$ \\
HIVIASI9 & HIV (58) & ASI9 $(12)$ \\
HIVIASI5 & HIV (23) & ASI5 (23) \\
HIVIASII & HIV (23) & ASII (33) \\
HIVIAS6 & HIV (II) & AS6 (I6) \\
HIV/RI & HIV (52) & HIV/RI $(>62)$ \\
ASI9/RI & ASI9/RI $(>62)$ & ASI9/RI $(>62)$ \\
\hline
\end{tabular}

a the same virus mix was tested on PBMC and SupTI cells

$b$ the day of outgrowth (>90\%) is indicated between brackets; the experiment has been repeated five times for ASI 9 and two times for the other variants

lhNef mediated inhibition of HIV-1 is specific and not due to non-specific effects, e.g. due to IFN induction by dsRNA.

\section{Conclusion}

In this study, we show that HIV-1 expressing an excessively stable $300 \mathrm{bp}$ lhNef hairpin potently inhibits wildtype HIV-1 in trans. However, HIV-lhNef did not replicate to detectable levels in HIV-1 target cells, probably because steps of its replication cycle are affected by the hairpin insertion, e.g. RNA splicing, RNA nuclear export or mRNA translation. Moreover, HIV-lhNef may cause degradation of its own mRNA due to processing by the RNAi machinery. We postulate that the lhNef hairpin may induce an antiviral response against wild-type HIV-1 either through a sequence-specific RNAi mechanism or through aberrant HIV-1 transcripts lacking the 3 ' non-coding region and polyA tail that induce a potent HIV-1-specific RNA silencing response in cells. Tests with reporter constructs do not support a sequence-specific RNAi effect, although the effect induced by lhNef is HIV-1 specific. The mechanism of trans-inhibition is currently unknown, but it remains of interest to study the molecular details because the observed inhibition is extremely potent. We are currently dissecting the mechanism of inhibition by testing HIVlhNef variants that lack important RNA signals (TAR, polyA, PAS, PBS, DIS, SD, psi).

Although HIV-lhNef is a potent inhibitor of wild-type HIV-1, its inability to replicate precluded long-term inhibition experiments. After prolonged culturing of HIVlhNef, replicating variants emerged through recombination events that introduce large truncations in the lhNef hairpin structure and therefore deletions in the Nef gene. Interestingly, all AS variants inherit a part of the hairpin structure in their genome, there were no perfect deletions of the entire lhNef region. One could speculate that the remaining secondary RNA structures are actively selected because they render the viral genome less susceptible for degradation by the RNAi machinery [11].
Nef represents a pathogenicity factor that disorders adaptive immunity by down regulating CD4 and MHC-1 receptors, by inhibiting T-cell chemotaxis, and by inducing apoptosis in bystander T-cells, and hence plays a major role in the destruction of the host immune system $[32,33]$. It has been suggested that any therapeutic intervention aimed at either completely blocking or at least partially reducing the expression of Nef during HIV-1 infection would likely enhance the ability of the immune system to fight HIV infection [34]. Humans infected with Nef-defective HIV-1 strains show low viral loads and no or very slow disease progression and represent long-term non-progressors or long-term slow-progressors $[31,35]$. Moreover, macaques vaccinated with a SIV strain that only lacks Nef are better protected against superinfection than macaques vaccinated with a SIV strain lacking the three accessory genes Nef, Vpr and Vpx [36]. The cross-protection conferred by the attenuated SIV strains appears not to be based on stimulation of the adaptive immune system, but on other (unknown) mechanisms [37]. In this study, we demonstrate effective outgrowth of the Nef-minus AS escape variants in competitions with wild-type HIV-1. This result was obtained in the SupT1 T cell line, which does not provide a clear Nef-phenotype. We think that this outcompetition is related to the presence of the genomic hairpin structure, because no such effect was observed for the control Nef-deletion virus R1. These in vitro results may relate to the superior protection with Nef-deleted viruses in vivo.

Successful expression of a shRNA from a replicating viral vector has been shown for Rous sarcoma virus (RSV) in avian cells [38]. We previously described inhibition of HIV-1 replication by a conditional-live HIV-rtTA virus that expresses a shRNA against the Nef gene [27]. This approach is especially suitable for targeting cells that are susceptible to HIV-1 infection. However, HIV-1 escape variants will emerge rapidly under shNef pressure [9]. Expression of multiple shRNAs or a single lhRNA from such a vector could prevent viral escape because multiple 


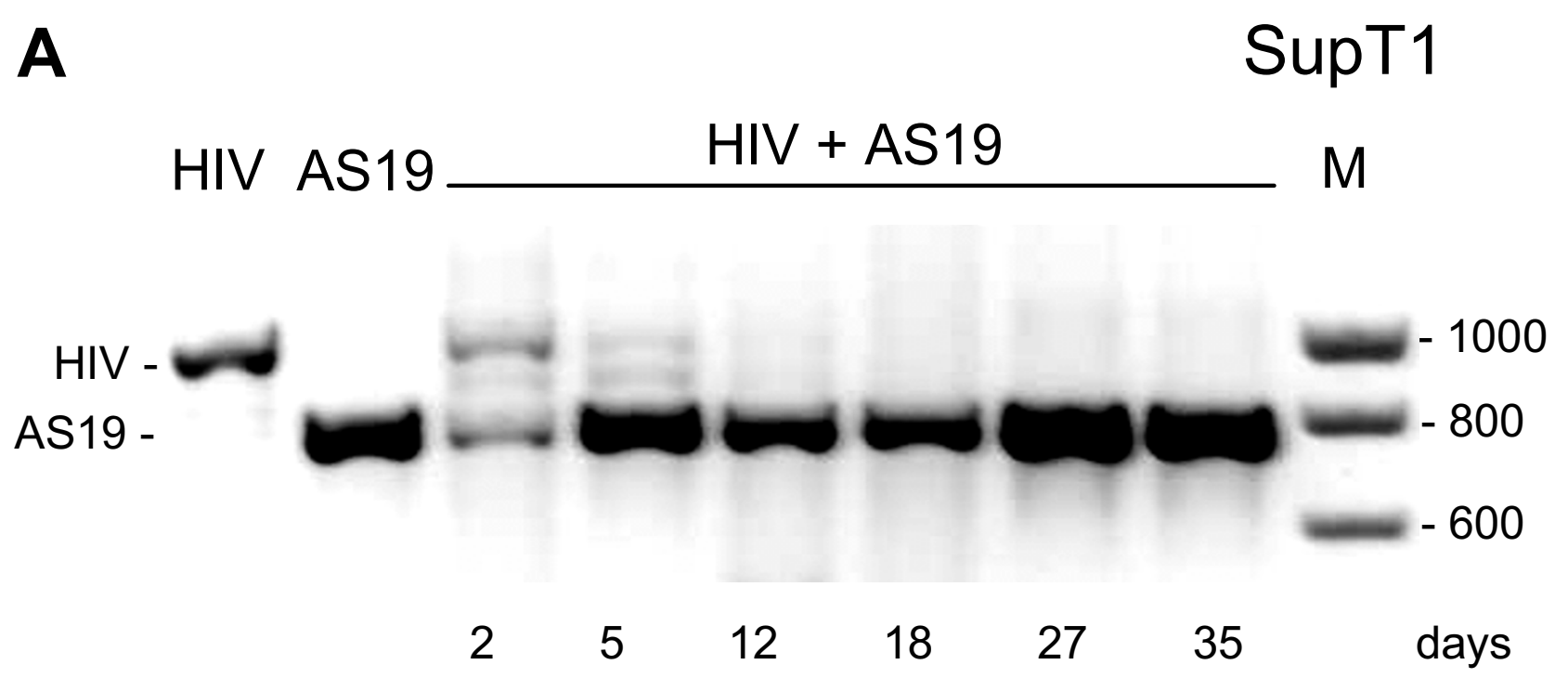

B

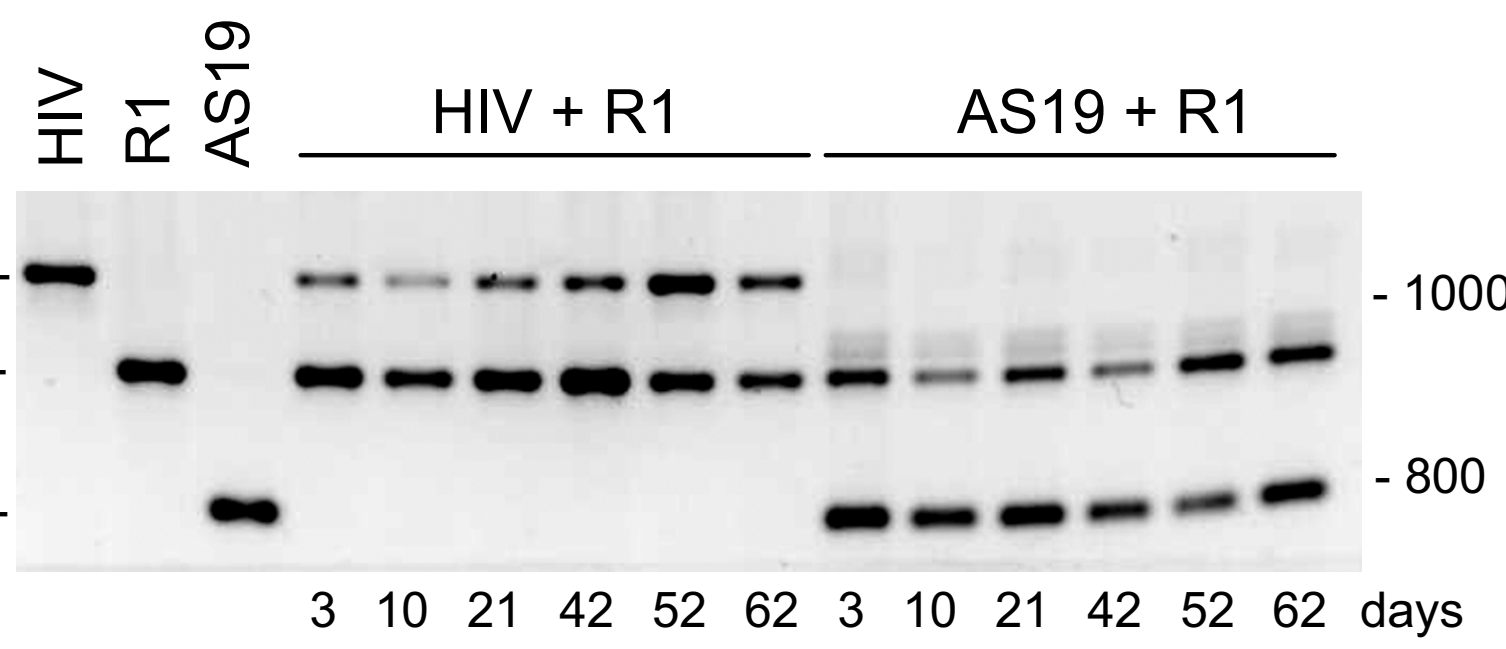

Figure 6

Virus competition experiments in the SupTI T cell line. See legend to Fig. 5 for details. (A) Competition between HIV-I and ASI9. (B) Competition between the RI variant and either HIV-I or ASI9.

precise mutations should occur. Use of a murine leukemia virus (MLV) can also be used for efficient and stable delivery of anti-HIV-1 shRNA [39]. However, MLV can replicate only in actively dividing cells, which limits its application as a therapeutic virus.

One ideal property of replicating HIV-1-based viral vectors is that they specifically target HIV-1 susceptible cells. The replication-competent AS escape variants lost the trans-inhibitory properties of HIV-lhNef, but effectively outcompeted wild-type HIV-1 in T cells. It is important to asses what is the minimum length of a hairpin that can mediate trans-inhibition of wild type HIV-1. We have previously shown that a 19 nt shRNA expressed from conditionally replicating HIV-1-based virus, can inhibit viral replication [27]. Any hairpin longer than that should in theory mediate trans-inhibition of HIV-1. Further research is needed to see if we can design constructs that are replication competent, yet remain a potent trans-inhibitor of HIV-1. For example, a lhRNA variant with G-U wobbles could be designed, which will destabilize the RNA structure and therefore stimulate viral replication. Such an approach using 90-100 bp lhRNA molecules has been suggested as a means for intracellular immunisation 

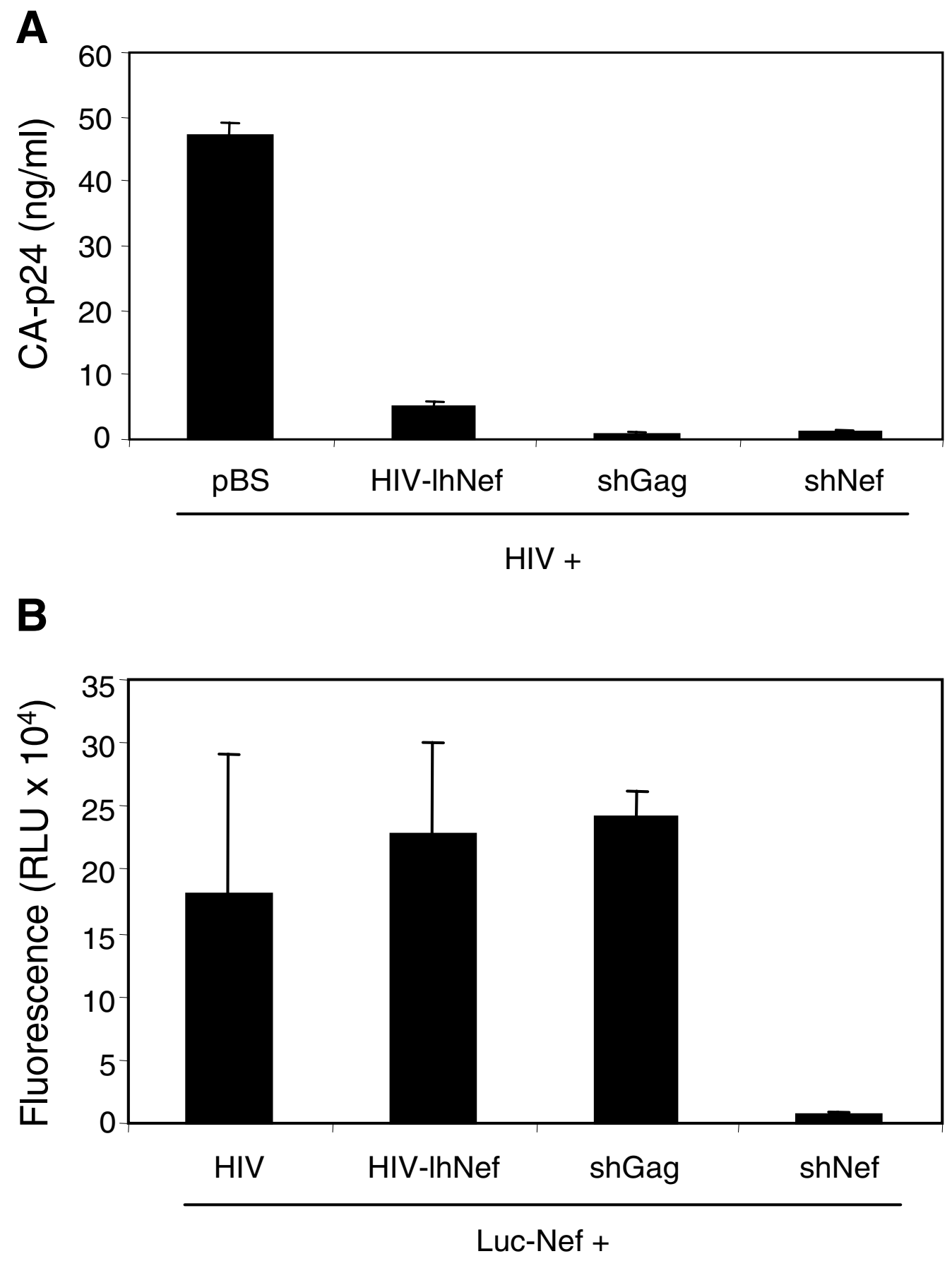

Figure 7

Test of sequence-specificity of the inhibition by HIV-lhNef. (A) Trans-inhibition of HIV-I production by HIV-IhNef and the RNAi-inducing shGag or shNef constructs. HEK293T cells were co-transfected with $100 \mathrm{ng} H I V-\mathrm{I}$ and I00 ng pBluescript, I00 ng HIV-IhNef, shGag or shNef. CA-p24 production was measured in the culture supernatant at two days post-transfection. Error bars represent the standard deviation in two independent experiments. (B) Inhibition of the Luc-Nef reporter. HEK 239T cells were co-transfected with $100 \mathrm{ng}$ of Luc-Nef and $100 \mathrm{ng}$ HIV-I, HIV-lhNef, shGag or shNef. Error bars represent the standard deviation in two independent experiments. 
against $\mathrm{HBV}$ and $\mathrm{HCV}$, without evoking non-specific IFN responses $[19,40]$. Such constructs could form the basis for an attenuated virus vaccine and anti-HIV therapeutic virus in one.

\section{Materials and methods DNA constructs and proviral DNA analysis}

The full-length molecular HIV-1 clone LAI [41] (Accession number AF33819.3) was used to produce wild-type virus. HIV-lhNef has a $93 \mathrm{nt}$ deletion in the 5 ' region of $\mathrm{Nef}$ and a 300 bp inverted repeat within the Nef gene (Fig. 1). The construction of the HIV-lhNef mutant and the generation of molecular clones of HIV-lhNef escape virus variants AS44, AS19, AS15, AS11 and AS8 has been described previously [28]. The HIV-1 mutant R1 has a $106 \mathrm{nt}$ deletion in Nef $(+8513$ to +8619$)$ and has been described previously [9]. HIV-CMV and HIV-asCMV were constructed by inserting the CMV promoter in sense or antisense orientation at position +8069 of the LAI genome. pSuper-shGag and pSuper-shNef with the $\mathrm{H} 1$ polymerase III promoter have been described previously [13]. Nucleotide numbers refer to the position on the genomic HIV-1 RNA transcript, with +1 being the capped G residue. Plasmid pGL3Nef (Luc-Nef), in which 250 nt from the Nef gene was placed downstream of the Photinus luciferase reporter gene has been described previously [11].

For cellular DNA isolation, cells were pelleted for $4 \mathrm{~min}$ at $4000 \mathrm{rpm}$ and solubilized in $150 \mu \mathrm{l}$ lysis buffer $(10 \mathrm{mM}$ Tris-HCl pH 8.0, 1 mM EDTA, $0.5 \%$ Tween 20) and 200 $\mu \mathrm{g} / \mathrm{ml}$ proteinase $\mathrm{K}$ (Roche) at $56^{\circ} \mathrm{C}$ for $1 \mathrm{~h}$ and at $95^{\circ} \mathrm{C}$ for $10 \mathrm{~min}$. Proviral DNA sequences were PCR amplified from $5 \mu \mathrm{l}$ cellular lysate using the 5' Env primer tTA1-AD $(+8269$ to +8289 , ACA GCC ATA GCA GTA GCT GAG) and 3' U5 primer CN1 (+9283 to +9253, GGT CTG AGG GAT CTC TAG TTA CCA GAG TC) (Fig. 1). PCR amplification was performed in a $50 \mu \mathrm{l}$ reaction containing $1 \times$ PCR amplification buffer (Invitrogen), $0.5 \mathrm{mM} \mathrm{MgCl}_{2}, 25$ pmol of each primer, $0.2 \mathrm{mM}$ dNTPs and 2.5 units of AmpliTaq DNA polymerase (Perkin Elmer Applied Biosystem). The PCR program was as follows: $95^{\circ} \mathrm{C}$ for 5 min, 30 cycles of $30 \mathrm{sec}$ at $94^{\circ} \mathrm{C}, 30 \mathrm{sec}$ at $55^{\circ} \mathrm{C}, 30 \mathrm{sec}$ at $72^{\circ} \mathrm{C}$, and a final extension for $7 \mathrm{~min}$ at $72^{\circ} \mathrm{C}$. The PCR products were separated on a $1 \%$ agarose gel stained with ethidium bromide and compared to a standard DNA size marker (Eurogentec). HIV-lhNef could not be amplified with this PCR due to the extended inverted repeat that may form a cruciform DNA structure. The RNA structures formed by HIV-lhNef and the AS escape variants were predicted with the Mfold program [42].

\section{Cells, DNA transfection and virus infection}

Human embryonic kidney (HEK) 293T cells were grown as a monolayer in DMEM (Invitrogen) supplemented with $10 \%$ FCS, minimal essential medium nonessential amino acids, penicillin $(100 \mathrm{U} / \mathrm{ml})$ and streptomycin $(100 \mu \mathrm{g} / \mathrm{ml})$ at $37^{\circ} \mathrm{C}$ and $5 \% \mathrm{CO}_{2}$. One day before transfection, cells were trypsinized, resuspended in DMEM and seeded in 24-well plates at a density of $1.5 \times 10^{5}$ cells per well. Cells were co-transfected with 100 - 500 ng DNA with Lipofectamine 2000 (Invitrogen) according to the manufacturer's instructions. 1 ng pRL plasmid (Promega), expressing Renilla luciferase (RL) from the CMV promoter, was added as an internal control for cell viability and transfection efficiency. In all transfection experiments we have controlled for the DNA input by adding pBluescript (Promega) to reach identical DNA concentrations.

Peripheral blood mononuclear cells (PBMCs) were isolated from buffy coats by standard Ficoll-Hypaque density centrifugation, activated with phytohemagglutinin $(3 \mu \mathrm{g} /$ $\mathrm{ml}$ ), and cultured in complete RPMI 1640 medium (Invitrogen) with IL-2 $(100 \mathrm{U} / \mathrm{ml})$. Cells $\left(5 \times 10^{6}\right)$ were transfected with $10 \mu \mathrm{g}$ of the proviral DNA by electroporation in $250 \mu \mathrm{l} \mathrm{RPMI}$ with $20 \%$ FCS in $0.4-\mathrm{cm}$ cuvettes at $250 \mathrm{~V}$ and $960 \mu \mathrm{F}$, and $1 \times 10^{6}$ fresh cells and $5 \mathrm{ml}$ complete RPMI with IL-2 were added afterwards. PBMCs were infected with a fixed amount (5 ng CA-p24) of 293T-produced virus and maintained for up to 4 weeks. Half of the culture medium was replaced every 4 days by fresh complete RPMI 1640 medium with IL-2 and freshly activated PBMC $\left(2 \times 10^{6}\right)$ were added at every $2^{\text {nd }}$ addition.

The human T cell line SupT1 was cultured in RPMI 1640 medium supplemented with $10 \%$ fetal calf serum (FCS) (Hybond), penicillin (100 U/ml) and streptomycin (100 $\mu \mathrm{g} / \mathrm{ml})$ at $37^{\circ} \mathrm{C}$ and $5 \% \mathrm{CO}_{2}$. Cells were cultured in 25 $\mathrm{cm}^{2}$ flasks and split 1 to 10 twice a week. Cells were infected with equal amounts (5 ng CA-p24) of 293T-produced virus. When HIV-induced cytopathic effects were observed, virus replication was maintained by passage of the cell-free culture supernatant onto uninfected SupT1 cells. At each passage, cell and supernatant samples were stored at $-70^{\circ} \mathrm{C}$. Virus spread was followed by CA-p 24 ELISA on the culture supernatant as described previously [43] and Renilla expression was measured with the Renilla luciferase assay system (Promega).

\section{Additional material}

\section{Additional File 1}

Virus competition experiments in SupT1 and PBMC. The composition of the wild type HIV-1 and the AS escape mutants was followed by PCR across the Nef region with primers tTA1 and CN1 (see Fig. 1). Click here for file

[http://www.biomedcentral.com/content/supplementary/17424690-4-15-S1.ppt] 


\section{Additional File 2}

Graphic quantification of the relative viral abundance in SupT1 and PBMC. The density of the PCR products from Figures 5 and 6 were calculated with the ImageJ software.

Click here for file

[http://www.biomedcentral.com/content/supplementary/17424690-4-15-S2.ppt]

\section{Additional File 3}

Graphic quantification of the relative viral abundance in SupT1. The density of the PCR products from Additional file 1 were calculated with the ImageJ software.

Click here for file

[http://www.biomedcentral.com/content/supplementary/17424690-4-15-S3.ppt]

\section{Acknowledgements}

HIV-I RNA research in the Berkhout lab is sponsored by ZonMw (VICl grant), NWO-CW (TOP grant) and Senter-NOVEM (TS grant with Viruvation). The authors thank Stephan Heynen for performing CA-p24 Elisa and Nienke Westerink for providing the PBMC.

\section{References}

I. Haasnoot PCJ, Cupac D, Berkhout B: Inhibition of virus replication by RNA interference. J Biomed Sc 2003, 10:607-616.

2. Berkhout $B$, Haasnoot I: The interplay between virus infection and the cellular RNA interference machinery. FEBS Lett 2006, 580:2896-2902.

3. Capodici J, Kariko K, Weissman D: Inhibition of HIV-I infection by small interfering RNA-mediated RNA interference. J Immunol 2002, 169:5196-520I.

4. Jacque JM, Triques K, Stevenson M: Modulation of HIV-I replication by RNA interference. Nature 2002, 41 8:435-438.

5. Novina CD, Murray MF, Dykxhoorn DM, Beresford PJ, Riess J, Lee SK, Collman RG, Lieberman J, Shankar P, Sharp PA: siRNA-directed inhibition of HIV-I infection. Nat Med 2002, 8:68I-686.

6. Park WS, Miyano-Kurosaki N, Hayafune M, Nakajima E, Matsuzaki T, Shimada F, Takaku H: Prevention of HIV-I infection in human peripheral blood mononuclear cells by specific RNA interference. Nucleic Acids Res 2002, 30:4830-4835.

7. Anderson J, Banerjea A, Akkina R: Bispecific short hairpin siRNA constructs targeted to CD4, CXCR4, and CCR5 confer HIVI resistance. Oligonucleotides 2003, | 3:303-312.

8. Boden D, Pusch O, Silbermann R, Lee F, Tucker L, Ramratnam B: Enhanced gene silencing of HIV-I specific siRNA using microRNA designed hairpins. Nucleic Acids Res 2004, 32: II $154-1158$.

9. Das AT, Brummelkamp TR, Westerhout EM, Vink M, Madiredjo M, Bernards R, Berkhout B: Human immunodeficiency virus type I escapes from RNA interference-mediated inhibition. J Virol 2004, 78:260I-2605

10. Boden D, Pusch O, Lee F, Tucker L, Ramratnam B: Human immunodeficiency virus type I escape from RNA interference. J Virol 2003, 77:11531-II535.

II. Westerhout EM, Ooms M, Vink M, Das AT, Berkhout B: HIV-I can escape from RNA interference by evolving an alternative structure in its RNA genome. Nucleic Acids Res 2005, 33:796-804.

12. Berkhout $B$ : RNA interference as an antiviral approach: targeting HIV-I. Curr Opin Mol Ther 2004, 6: I4I-I45.

13. Ter Brake O, Konstantinova P, Ceylan M, Berkhout B: Silencing of HIV-I with RNA Interference: a multiple shRNA approach. Mol Ther 2006, I 4:883-892.

14. Ter Brake O, Berkhout B: A novel approach for inhibition of HIV-I by RNA interference: counteracting viral escape with a second generation of siRNAs. Journal of RNAi and Gene Silencing 2005, I(2):56-65.
15. Yeung ML, Bennasser Y, Myers TG, Jiang G, Benkirane M, Jeang KT: Changes in microRNA expression profiles in HIV-I-transfected human cells. Retrovirology 2005, 2:8I.

16. Hariharan M, Scaria V, Pillai B, Brahmachari SK: Targets for human encoded microRNAs in HIV genes. Biochem Biophys Res Commun 2005, 337:1214-1218.

17. Yamamoto T, Omoto S, Mizuguchi M, Mizukami H, Okuyama H, Okada N, Saksena NK, Brisibe EA, Otake K, Fuji YR: Doublestranded nef RNA interferes with human immunodeficiency virus type I replication. Microbiol Immunol 2002, 46:809-8I7.

18. Konstantinova P, de Vries W, Haasnoot J, Ter Brake O, de Haan P, Berkhout B: Inhibition of human immunodeficiency virus type I by RNA interference using long-hairpin RNA. Gene Ther 2006, I3:1403-14|3.

19. Akashi H, Miyagishi M, Yokota T, Watanabe H, Hino T, Nishina $\mathrm{K}$, Kohara M, Taira K: Escape from the interferon response associated with RNA interference using vectors that encode long modified hairpin-RNA. Molecular BioSystems 2005, I:382-390.

20. Matsumoto S, Miyagishi M, Akashi H, Nagai R, Taira K: Analysis of double-stranded RNA-induced apoptosis pathways using interferon-response noninducible small interfering RNA expression vector library. J Biol Chem 2005, 280:25687-25696.

21. Nishitsuji H, Kohara M, Kannagi M, Masuda T: Effective suppression of human immunodeficiency virus type I through a combination of short- or long-hairpin RNAs targeting essential sequences for retroviral integration. J Virol 2006, 80:7658-7666.

22. Elbashir SM, Lendeckel $W$, Tuschl T: RNA interference is mediated by 2I- and 22-nucleotide RNAs. Genes Dev 200I, 15:188-200.

23. Caplen NJ, Zheng Z, Falgout B, Morgan RA: Inhibition of viral gene expression and replication in mosquito cells by dsRNA-triggered RNA interference. Mol Ther 2002, 6:243-25I.

24. $\mathrm{Q} i \mathrm{Y}$, Zhong $X$, Itaya $A$, Ding $B$ : Dissecting RNA silencing in protoplasts uncovers novel effects of viral suppressors on the silencing pathway at the cellular level. Nucleic Acids Res 2004, 32:el79.

25. Waterhouse PM, Graham MW, Wang MB: Virus resistance and gene silencing in plants can be induced by simultaneous expression of sense and antisense RNA. Proc Natl Acad Sci USA 1998, 95:13959-13964.

26. Adelman ZN, Sanchez-Vargas I, Travanty EA, Carlson JO, Beaty BJ, Blair $C D$, Olson KE: RNA silencing of dengue virus type 2 replication in transformed $\mathrm{C} 6 / 36$ mosquito cells transcribing an inverted-repeat RNA derived from the virus genome. J Virol 2002, 76: I2925-12933.

27. Westerhout EM, Vink M, Haasnoot PC, Das AT, Berkhout B: A conditionally replicating HIV-based vector that stably expresses an antiviral shRNA against HIV-I replication. Mol Ther 2006, 14:268-275.

28. Konstantinova $P$, de Haan $P$, Das AT, Berkhout B: Hairpin-induced tRNA-mediated (HITME) recombination in HIV-I. Nucleic Acids Res 2006, 34:2206-2218.

29. Beerens N, Groot F, Berkhout B: Stabilization of the U5-leader stem in the HIV-I RNA genome affects initiation and elongation of reverse transcription. Nucleic Acids Res 2000, 28:4I30-4I37.

30. de Ronde A, Klaver B, Keulen W, Smit L, Goudsmit J: Natural HIVI NEF accelerates virus replication in primary human lymphocytes. Virol 1992, 188:391-395.

31. Deacon NJ, Tsykin A, Solomon A, Smith K, Ludford-Menting M, Hooker DJ, McPhee DA, Greenway AL, Ellett A, Chatfield C, Lawson VA, Crowe S, Maerz A, Sonza S, Learmont J, Sullivan JS, Cunningham A, Dwyer D, Dowton D, Mills J: Genomic structure of an attenuated quasi species of HIV-I from blood transfusion donor and recipients. Science 1995, 270:988-99।.

32. Swingler S, Brichacek B, Jacque JM, Ulich C, Zhou J, Stevenson M: HIV-I Nef intersects the macrophage CD40L signalling pathway to promote resting-cell infection. Nature 2003, 424:213-219.

33. Schiavoni I, Trapp S, Santarcangelo AC, Piacentini V, Pugliese K, Baur A, Federico M: HIV-I Nef enhances both membrane expression and virion incorporation of Env products. A model for the Nef-dependent increase of HIV-I infectivity. J Biol Chem 2004, 279:22996-23006. 
34. Yamamoto T, Miyoshi H, Yamamoto N, Yamamoto N, Inoue J, Tsunetsugu-Yokota $Y$ : Lentivirus vectors expressing short hairpin RNAs against the U3-overlapping region of HIV nef inhibit HIV replication and infectivity in primary macrophages. Blood 2006, 108:3305-3312.

35. Rhodes DI, Ashton L, Solomon A, Carr A, Cooper D, Kaldor J, Deacon N: Characterization of three nef-defective human immunodeficiency virus type I strains associated with long-term nonprogression. Australian Long-Term Nonprogressor Study Group. J Virol 2000, 74:1058I-10588.

36. Johnson RP: Live attenuated AIDS vaccines: hazards and hopes. Nature Medicine 1999, 5:I54-I55.

37. Stebbings R, Berry N, Stott J, Hull R, Walker B, Lines J, Elsley W, Brown S, Wade-Evans A, Davis G, Cowie J, Sethi M, Almond N: Vaccination with live attenuated simian immunodeficiency virus for 21 days protects against superinfection. Virol 2004, 330:249-260.

38. Bromberg-White JL, Webb CP, Patacsil VS, Miranti CK, Williams BO, Holmen SL: Delivery of short hairpin RNA sequences by using a replication-competent avian retroviral vector. J Virol 2004, 78:49|4-49|6.

39. Sliva K, Schnierle BS: Stable integration of a functional shRNA expression cassette into the murine leukemia virus genome. Virol 2006, 35 I:218-225.

40. Weinberg MS, Ely A, Barichievy S, Crowther C, Mufamadi S, Carmona S, Arbuthnot P: Specific Inhibition of HBV Replication In Vitro and In Vivo With Expressed Long Hairpin RNA. Mol Ther 2007, I 5(3):534-4I.

41. Peden K, Emerman M, Montagnier L: Changes in growth properties on passage in tissue culture of viruses derived from infectious molecular clones of HIV-ILAI, HIV-IMAL, and HIVIELI. Virol 199|, 185:661-672.

42. Zuker M: Mfold web server for nucleic acid folding and hybridization prediction. Nucleic Acids Res 2003, 31:3406-34I5.

43. Jeeninga RE, Hoogenkamp M, Armand-Ugon M, de Baar M, Verhoef $\mathrm{K}$, Berkhout B: Functional differences between the long terminal repeat transcriptional promoters of HIV-I subtypes $A$ through G. J Virol 2000, 74:3740-375 I [http://PM:10729|49].

\section{Publish with Bio Med Central and every scientist can read your work free of charge}

"BioMed Central will be the most significant development for disseminating the results of biomedical research in our lifetime. "

Sir Paul Nurse, Cancer Research UK

Your research papers will be:

- available free of charge to the entire biomedical community

- peer reviewed and published immediately upon acceptance

- cited in PubMed and archived on PubMed Central

- yours - you keep the copyright

Submit your manuscript here:

http://www.biomedcentral.com/info/publishing_adv.asp
BioMedcentral 